\title{
THE REALIZED AND EXPECTED EARLY CHILDHOOD INTERVENTION PRACTICES IN LITHUANIA
}

\author{
Stefanija Alisauskiene
}

Siauliai University, Lithuania

\begin{abstract}
Inclusive early childhood practices are at the forefront of the research and practice efforts in many European countries. Inclusion in early childhood programs can set a trajectory for inclusion across the life course, making it critical that we include individuals with special educational needs in all facets of society from birth. In many European countries, including Lithuania, children with special educational needs and their families continue facing significant barriers to accessing inclusive high-quality early childhood practices and too many pre-school children with special educational needs and disabilities continue receiving special education services in separate settings, as opposed to the least restrictive environment. The aim of the research was to assess the realized and expected early childhood intervention practices while educating children with diverse needs in inclusive pre-school settings. The study was based on a survey using the instruments of Recommended Practices in Early Intervention/Early Childhood Special Education. The instrument includes the following topic areas: leadership, assessment, environment, family, instruction, interaction, collaboration, and transition.
\end{abstract}

Keywords: children with special educational needs, early childhood intervention, educational support, family support.

\section{Introduction}

Inclusion is an educational approach and philosophy that provides all children with community membership and greater opportunities for achievements, particularly for social-emotional ones. Inclusion is about making sure that every child from his/her birth feels welcomed and that his/her unique needs and learning styles are recognized, valued, and met. This approach seems to be highly valuable in today's turbulent social economic and cultural contexts. An equitable inclusive education system is one in which all children, parents, other family, and community members are welcomed and respected, as well as every child is supported and inspired to succeed in the culture of high expectations for learning. The basic premise of inclusive education is that schools are about belonging, nurturing and educating all students regardless of their differences in ability, culture, gender, language, class and ethnicity (Kozleski et al., 2007). Educational settings and teachers, therefore, need to commit to the transformation of their communities for the implementation of inclusive education to be successful. Regarding the importance of the role of 
teachers, research indicates that they play a critical role in the implementation of inclusive education (Forlin et al., 2010). The stress on inclusion from early childhood means holding high expectations and intentionally promoting participation in all learning and social activities, facilitated by individualized accommodations, and using evidence-based services and supports to foster their development, as well as a sense of belonging and friendships with peers (Policy Statement on Inclusion, 2015). This applies to all children, including those with special educational needs (SEN). The majority of European countries implement inclusive education and focus on the meeting of the diverse educational needs of children ${ }^{1}$. Nevertheless, in many European countries, including Lithuania, children with SEN and their families continue facing significant barriers to access inclusive high-quality early childhood practices and too many pre-school children with SEN continue to receive special education services in separate settings.

The article focuses on inclusive early childhood education and intervention (ECEI) practices. The aim of the research was to assess the realized and expected ECEI practices while educating children with diverse needs in inclusive pre-school settings. The study was based on a survey Recommended Practices in Early Intervention/Early Childhood Special Education ${ }^{2}$. The article presents the data collected in Lithuania in 2015.

\section{The Concept of Inclusive Early Childhood Education}

We base our understanding of inclusive education on the internationally adopted definition which highlights that inclusive education is an ongoing process aimed at offering quality education for all while respecting diversity and the different needs and abilities, characteristics and learning expectations of the students and communities, eliminating all forms of discrimination (UNESCO, 2009). The philosophy of educating children gradually focused on providing equal educational opportunities from a rights-based perspective which had led to inclusive education continuing to be implemented in most European countries and beyond over the past three decades. Inclusive education has increasingly become a focus of debate in discussions about the development of the educational policy and practice around the world (Farrell \& Ainscow, 2002). Regarding the practicality of fully implementing inclusive education, many jurisdictions, though, continue to be reluctant to support inclusion (Woolfson \& Brady, 2009; Alghazo \& Gaad, 2004), especially when learners have more challenging support needs (Talmor, Reiter \& Feigin, 2005). However, the

\footnotetext{
${ }^{1}$ www.european-agency.org

${ }^{2}$ DEC/Division for Early Childhood (2014). Recommended Practices in Early Intervention/Early Childhood Special Education 2014. Retrieved from http: //www.dec-sped.org/recommendedpractices.
} 
education of children with SEN is now an established key policy objective in many countries. Lithuania follows the description of children with SEN as having a need for assistance and services in the education process that occurs due to being exceptionally gifted, having congenital or acquired disorders or disadvantages in a person's surroundings (Teacher Education for Inclusion, 2010). Equity and inclusive education aims to understand, identify, address, and eliminate the biases, barriers, and power dynamics that limit children prospects for learning, growing, and fully contributing to society (Equity \& Inclusive Education, 2014). Teachers, school administrations, school boards and policies do emphasize the need to achieve an inclusive classroom atmosphere. However, it is striking even over a decade into the unfolding of such policies, how tenuously these efforts are perceived as having any tangible links with the human rights dimension and the fundamental imperative to provide each student with access to learning (Beach, 2010). Accessibility in ECEI programs means that all children can interact with materials, activities, teachers, and peers to the fullest extent possible and with equal frequency and enjoyment. When applied in ECEI settings, universal design concepts refer to the program's physical features as well as to such features as daily schedule and classroom routines, curriculum, and teaching strategies (Conn-Powers et al., 2006; Klein et al., 2000). We base our understanding of inclusion in ECEI as holding high expectations and intentionally promoting participation of children in all learning and social activities, facilitated by individualized accommodations, and using evidencebased services and supports to foster their development, friendships with peers and sense of belonging. This applies to all children including those with SEN. Supporting all children to fully participate in their communities requires highquality ECEI policies and practices. High-quality environment and support to classroom staff must characterize inclusive ECEI settings to ensure desirable outcomes for all children, especially those with SEN (Cate et al., 2010). Highquality inclusive ECEI consist of three key components: accessible to all children and their families; designed and carried out to show a great consideration for the unique needs of each child; ensure full participation (Underwood \& Frankel, 2012). In high-quality ECEI programs, all children have opportunities to develop their language, social, physical and cognitive abilities. Inclusive ECEI is not just about placement in a program, but also active participation in social interactions and the development of children's abilities and skills. Children at a range of developmental levels, including those with identified SEN, should be welcomed as valued members of ECEI community by supporting active participation (ibid). Parents are among the most influential yet significantly underrated actors in their children's education. By recognizing parents' roles in their child's education, and working together, staff and parents can create and maintain a high-quality education for children. Professionals 
working with young children and their families must recognize and respect their cultures, ethnicities, languages, values, faiths, and belief systems, and thus be able to effectively support children's development and learning. Cooperation between ECEI staff and parents is considered as beneficial for children's progress and development (Key Data on Education, 2009). Expertise from a wider community may help to improve the effectiveness of settings, ensure better use of resources and enabling extra-curricular activities to be delivered. For these reasons, many countries encourage ECEI practitioners to work closely with their stakeholders to support children's development, learning and general well-being (Key Data on ECEC in Europe, 2014). Inclusive education, as outlined in the EU documents, is a response to the widespread social, economic, political changes in Europe, as well as a means of establishing a caring, humane and egalitarian society. The introduction of this policy will, however, require extensive changes in ECEI, as the focus shifts from children's adjustment to the demands of the system, to the system's capability to accommodate all learners' needs as inclusively as possible.

\section{Inclusive Early Childhood Practices in Lithuania: Realized and Expected}

\section{Research Design}

Research methodology is based on the concept of inclusive education and the methodological approach, which combines exploratory and descriptive research. The exploratory research, which is broad in focus, helped us identify the key issues related to realized and expected ECEI practices. Descriptive research used to accurately assess and describe the realized and expected inclusive ECEI practices while educating young children with SEN in preschool settings in Lithuania. For this survey, the data has been collected via online questionnaires 'Recommended Practices in Early Intervention'. The article presents a part of the complex research data, taking into account the comments of participants. The data has been collected in Lithuania in the period August to November, 2015. There were 280 questionnaires collected (215 valid for analysis). The group of respondents consisted of 56 pre-school teachers, 47 speech therapists, 50 coordinators of the Child Welfare Commission, 27 other specialists, 14 special pedagogues, 8 social pedagogues, 7 psychologists, 3 social pediatricians, 1 social worker, 1 neurologist, 1 physiotherapist; 65 respondents omitted this information. The vast majority of respondents were female, and only two males took part. The respondents represented 149 preschool settings, 10 early childhood intervention services, 37 other institutions related to ECEI. All settings included children with SEN. 
Ethical issues have been considered when conducting the research. The participants received information about the research aims, methods, and the use of the results online before the research started. Confidentiality of their personalities has been assured. In order to respect the privacy and autonomy of the specialists, only these who were willing took part in the research.

\section{Results}

\section{Realized and Expected Inclusive Early Childhood Practices}

The realized and expected ECEI practices have been explored and described taking into account the comments of participants on leadership, assessment, environment, family, instruction, interaction, collaboration, and transition.

Leadership. The work of practitioners at the frontline is critical to improve outcomes for young children who have, or are at risk for, developmental delays/SEN and their families, but practitioners do not operate in a vacuum. Leaders establish the conditions that are essential for the successful implementation of ECEI (DEC, 2014). The analysis of the comments on leadership (115 comments received) revealed that participants working in the field of ECEI stressed the leader's contribution to team work and educational environment as key issues realized in ECEI practice, while the most expected ones are to receive more resources (material, human, informational, time etc.) and to ensure improved collaboration with parents. According to the requirements, leaders should promote efficient and coordinated service delivery for children and families by creating the conditions for practitioners from multiple disciplines and the family to work together as a team. Therefore, respondents admitted that 'leaders create and implement policies and practices that promote the shared decision-making with practitioners and families, and attention is focused on how 'to adjust an educational environment for all children, particularly for those who have special needs'. On the other hand, they said 'no children with SEN - no problems'. Leaders meet a lot of challenges related to resources: 'it is difficult to find money to cover professionals training courses which are quite expensive', 'to engage more specialists', etc. According to respondents, the collaboration with parents is valued, but it is not sufficient because 'not all parents are collaborating, they used to conceal child's problems', etc. The personal characteristics of leaders and their efforts to create a positive microclimate in ECEI have been also highlighted. The results showed that leaders did much to create the culture and climate in which practitioners would feel a sense of belonging and want to support the organization's mission and goals ('leaders create a warm atmosphere in the kindergarten', 'everyone 
Stefanija Alisauskiene. The Realized and Expected Early Childhood Intervention Practices in Lithuania

can feel he/she is valued and important', etc.). However, some comments relate to 'autocratic style of management', 'stereotypical attitudes toward education and changes', etc. For the overall picture of realized and expected leadership see Fig. 1.

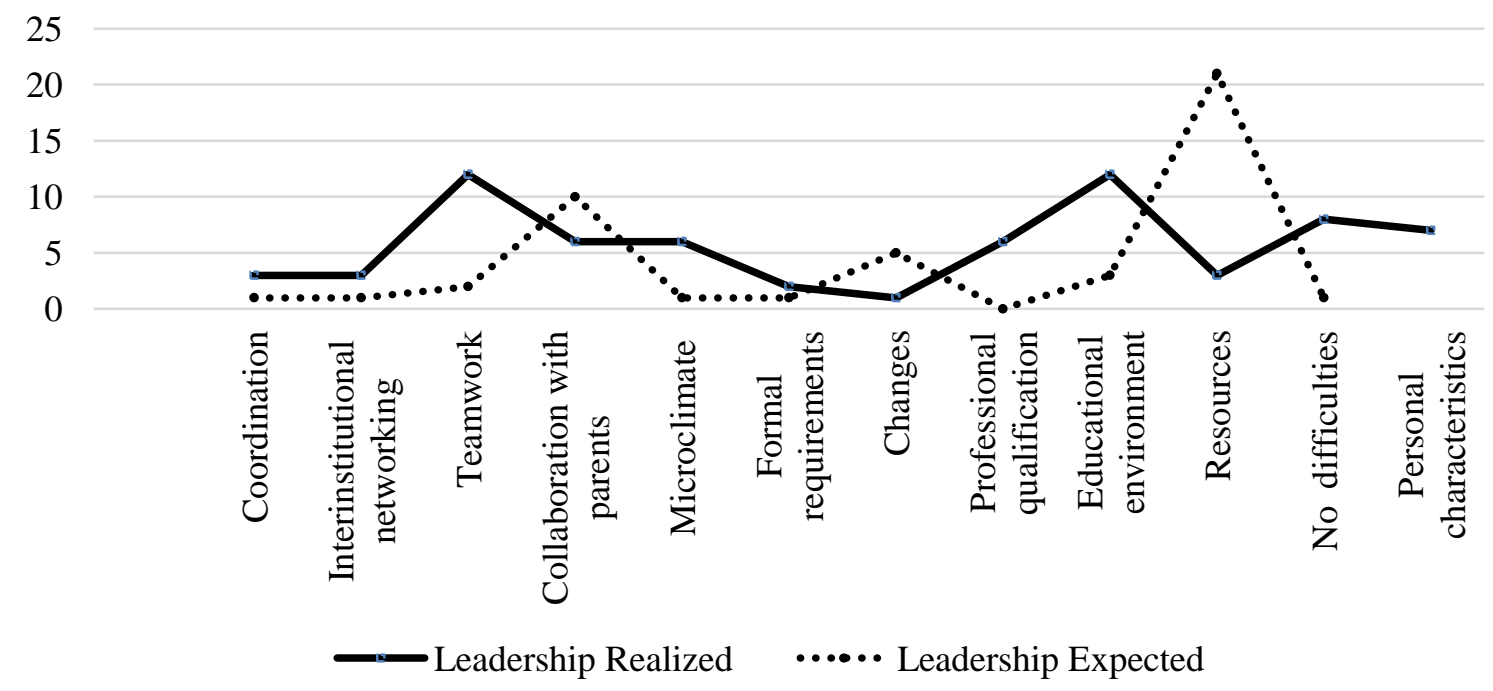

Fig. 1 Realized and expected ECEI practices related to leadership

Assessment in the field of ECEI is the process of gathering information to make decisions, and it is conducted for the purposes of screening, determining eligibility for services, individualized planning, monitoring child's progress, and measuring child's outcomes (DEC, 2014). The comments $(n=122)$ of the participants have been analyzed, and 8 categories related to the assessment have been identified: collaboration with parents, team work, use of assessment instruments, assessment for education and support, assessment competence, assessment as a process, strengths and needs focused assessment, and formal assessment. The results show that the assessment for education and support, the collaboration with parents and the teamwork have been introduced as the prior issues realized in their ECEI practices. Practitioners implement systematic ongoing assessment to identify learning targets, plan activities, and monitor child's progress to revise instruction as needed: 'we developed the system of assessment to identify peculiarities, needs, and social situation of the child'. It helps us develop an Individual Education/Support Plan'; 'assessment is a process which facilitates support to a child and a family', etc. According to the respondents, they collaborate with parents in their ECEI practice: 'the opinion of parents is important for us', 'the priorities of child's education are set up together with parents', 'cooperation with parents ensures the continuity of the support to a child'. However, it needs to be improved: 'information provided by parents is not always correct', 'we cannot always trust to them', 'not every 
family knows how to support their child', 'parents are very cautious about the assessment' etc. Also, the assessment for child education and support have been highlighted as requiring some improvement. According to the respondents, there is lack of specialists ('mostly the speech therapist is responsible for the needs' assessment'), and lack of competence to properly assess a young child ('this is difficult to assess a child due to his/her poor communication'). The key categories of the comments related to the assessment are displayed in Fig. 2.

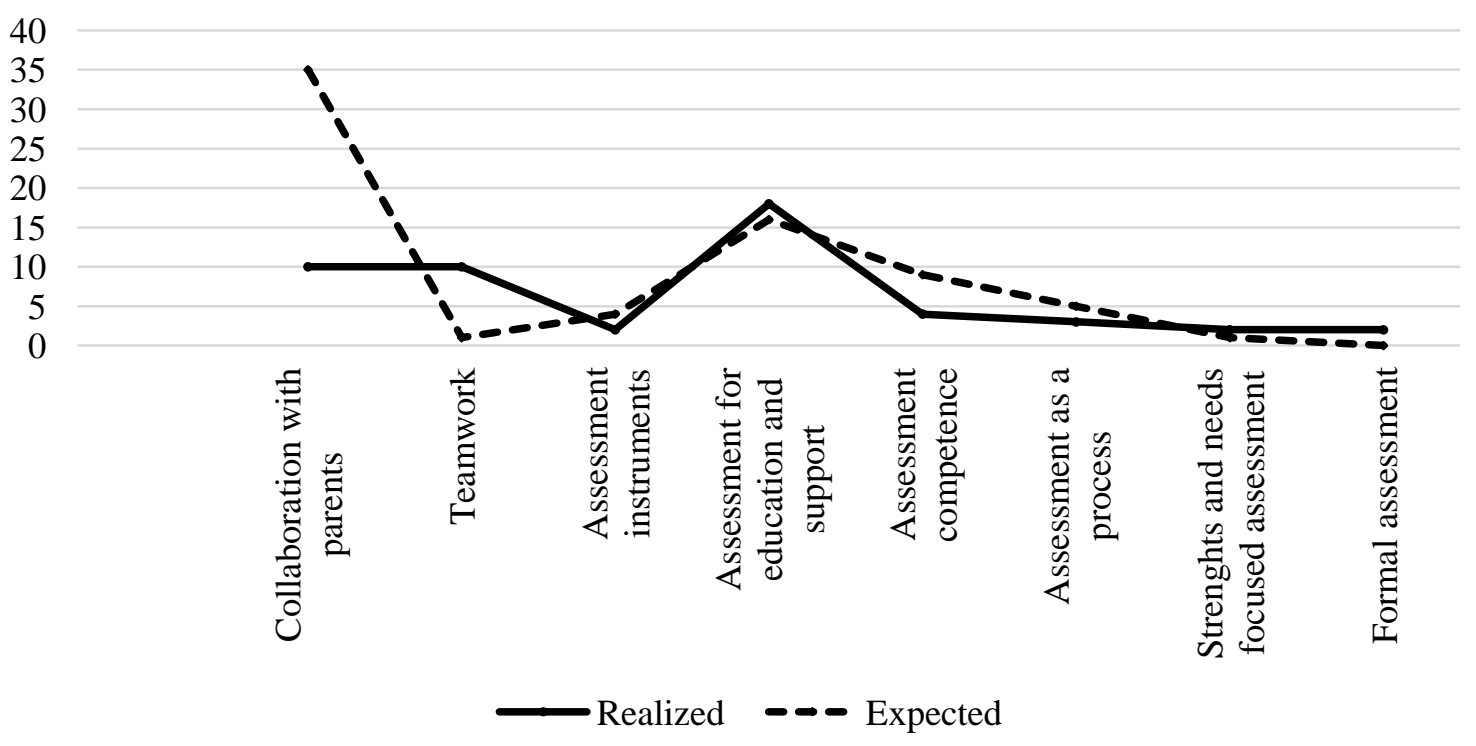

Fig. 2 Realized and expected ECEI practices related to assessment

Environment. Young children including those who have, or are at risk for, SEN learn, play, and engage with adults and peers within a multitude of environments such as home, school, child care, and the neighbourhood. Environmental practices refer to the aspects of space, materials (toys, books, etc.), equipment, routines, and activities that practitioners and families can intentionally alter to support each child's learning across developmental domains. Through the implementation of the environmental practices, practitioners and families can promote nurturing and responsive learning environments that can foster each child's development (DEC, 2014). The comments $(n=102)$ of the participants are focused on eight categories of realized and expected practices: environment adjusted to the age, safe environment, specialized environment, environment available for children with SEN; and lack of space, lack of professionals, overcrowded rooms, disagreements with parents. The analysis of the comments revealed that the environment was sufficiently adjusted to children's age, and it was at some degree available for children with SEN: 'the suitable educational environment for all children according to their age is created', 'there is a lot of extra space in the kindergarten', 'we have a 
special group of children with severe SEN. Those who have moderate SEN are integrated into general groups with their peers', 'we use specialised environment to provide special support to children, e.g. room of a speech therapist', etc. However, it is expected that environment should be much more available for children with SEN: 'we have no facilities in order to provide proper support to children with SEN', 'outdoor spaces are not adjusted'. On the other hand, there were some comments, which showed a clear relation between physical environment of the setting and the attitudes of professionals: 'we are not implementing inclusive education - we have no children with disabilities'. The evidence shows that parents and professionals have disagreements on environmental issues in ECEI settings: 'often the expectations of a family are higher than the school can meet'. Realized and expected practices related to environment are displayed in Fig. 3.

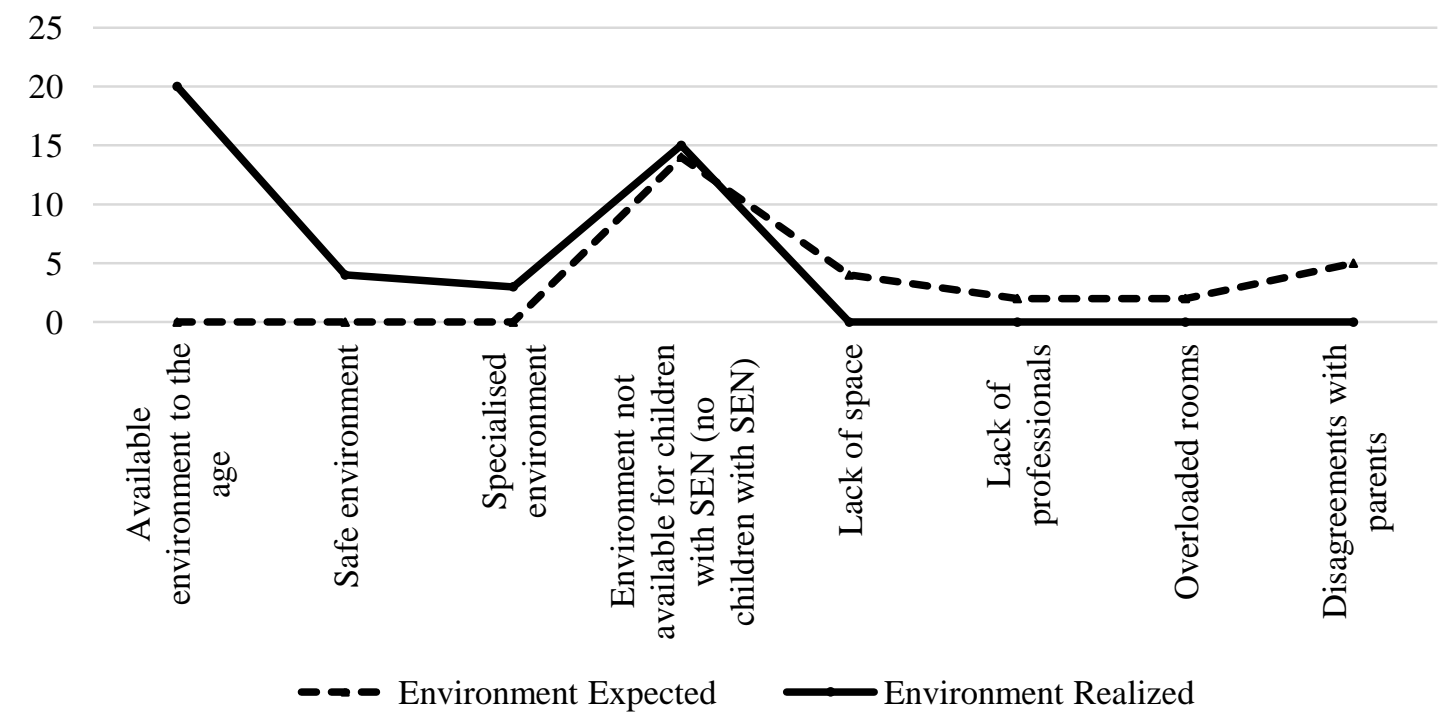

Fig. 3 Realized and expected ECEI practices related to environment

Family practices refer to ongoing activities that promote the active participation of families in the decision-making related to their child, lead to the development of a service plan, support families in achieving the goals they hold for their child. Family practices encompass three themes: family-centred and family capacity-building practices, family and professional collaboration (DEC, 2014). After analyzing the comments $(n=92)$ of the participants, we found out five categories on realized ECEI practices related to family, such as: professionals' initiative and competence, parents' initiative, resources, the briefing and educating of families, and the shift of responsibility. According to the participants, their initiative and professional competence to work with families together with their focus on training and information provision to families allow them realize ECEI practices: 'we have an excellent team of 
professionals including the Child Welfare Commission', 'specialists are searching for the means to cooperate with parents', 'we try to meet family expectations', 'we provide training for parents', 'we inform parents on child's achievements, provide recommendations for parents to support the child at home'. At the same time, participants expect much more parents' initiatives while educating young children and providing educational (including special) support to them: 'parents do not accept their child's disability', 'this is not easy to contact parents - they are always in a hurry, plunged in their routine', 'parents often don't know what they want', 'we, as a teachers don't want to disturb parents...'. Realized and expected practices related to family are demonstrated in Fig. 4.

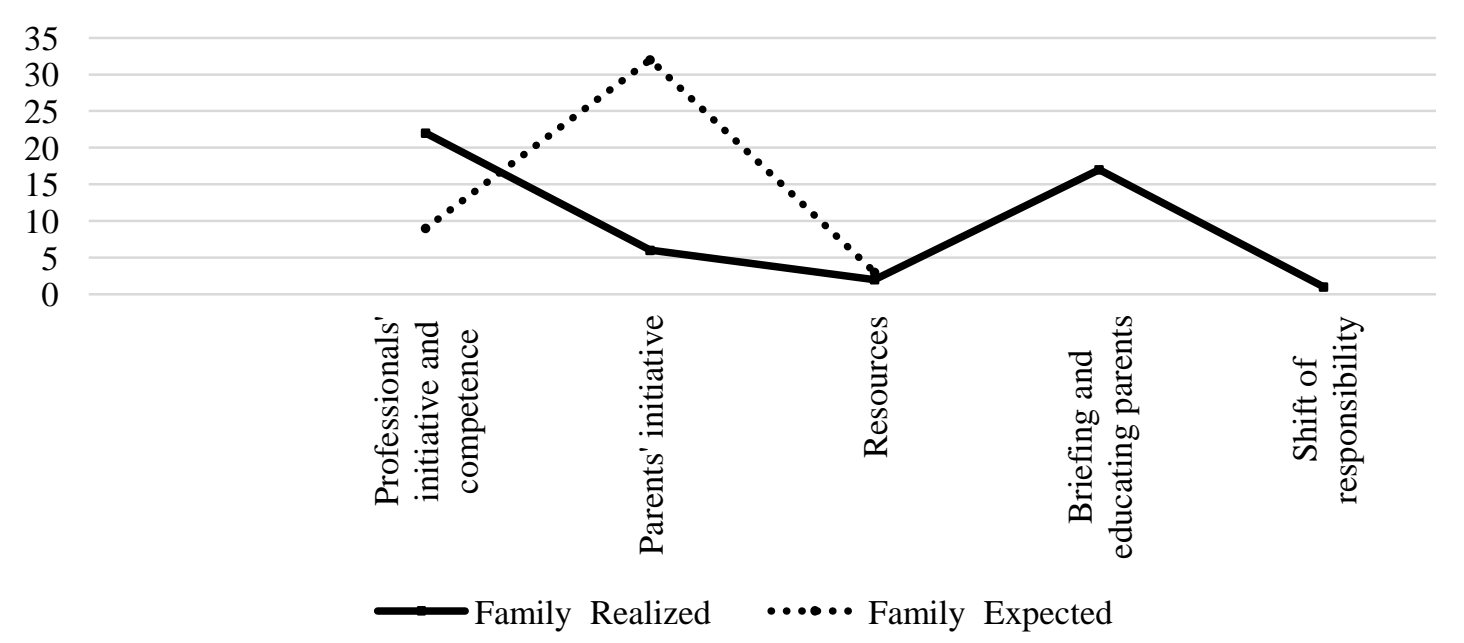

Fig. 4 Realized and expected ECEI practices related to family

Instructions. Instructional practices intentionally and systematically inform what and when to teach, how to evaluate the effects of teaching, how to support and evaluate the quality of instructional practices. Instructional practices are a subset of intervention activities conducted by practitioners and parents (DEC, 2014). The analysis of participants' comments $(n=99)$ revealed 5 key categories related to instruction: collaboration with parents, professional competences, the briefing and consulting of parents, teamwork, the individualized and timely support provided to children if needed. The results show that professional competence of instructions is recognized as well realized in ECEI practice: 'specialists observe, analyse, plan and provide support accordingly', 'they find different methods to involve every child into educational activities', 'we encourage children's participation', 'support is integrated into the routine'. On the other hand, participants expect it to be improved: 'kindergarten teachers lack of competence to educate children with moderate SEN, they don't pay 
Stefanija Alisauskiene. The Realized and Expected Early Childhood Intervention Practices in Lithuania

enough attention to these children', 'we provide support according to kindergarten's resources and potential, but this not always suits children's needs'. The evidences show, that ECEI proceeds in teamwork and tend to provide information / consultation to parents rather than collaborate with them: 'in order to achieve good results specialists communicate and collaborate with teachers', 'the Child Welfare Commission do a lot to support teachers meeting SEN of children', 'individual support to a child is provided', 'consultations to a family are ensured'. However, 'not all parents come to meet specialists', 'parents do not do their 'homework' according to specialists' tasks to support a child', 'it is difficult to involve parents into support because they think that specialists alone should do this'. Nevertheless, the ECEI practitioners stressed that there was lack of collaboration with parents and that it needed to be improved (Fig. 5).

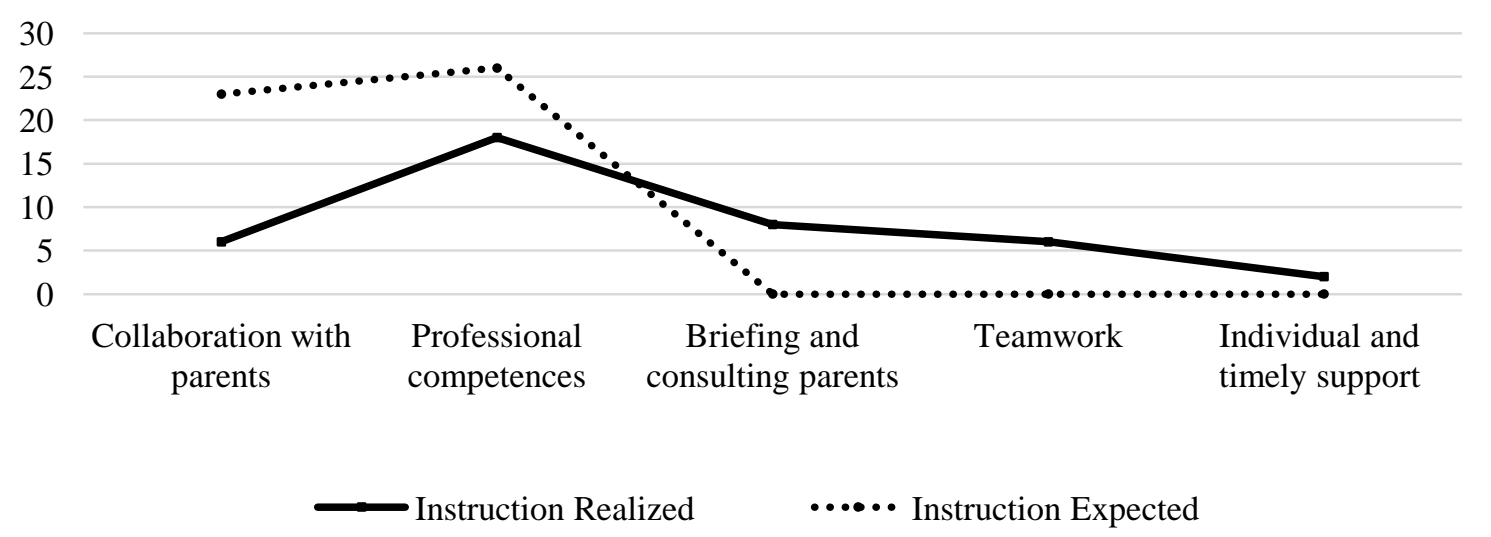

Fig. 5 Realized and expected ECEI practices related to instructions

Interaction. Sensitive and responsive interactional practices are the foundation for promoting the development of a child's language as well as cognitive and emotional competence. Practitioners engage in these practices across environments, routines, and activities and assist others in the child's life: family members, other caregivers, and peers, in learning sensitive and responsive ways to interact with the child and promote the child's development (DEC, 2014). The analysis of the comments $(n=73)$ showed that participants focused on interaction in order to identify child's needs, provide support, ensure positive home-institution relationships and child's relationship with peers: 'observation and communication with a child allows us recognize the main needs of the child', '...encourage child's motivation to communicate', 'nontraditional methods support child's ability to learn problem-solving skills', 'ensure the continuity of interaction in a kindergarten and at home', 'children 
with SEN are integrated into group activities with their peers', etc. On the other hand, specialists see the interaction as their duty: 'the teacher is responsible for everything, including unpredictable issues'. Results showed that through the interactions practitioners recognized children's needs, preferences, and interests, and promoted their development and education accordingly. Even though it is expected that interaction as a duty of pedagogues should be improved (Fig. 6).

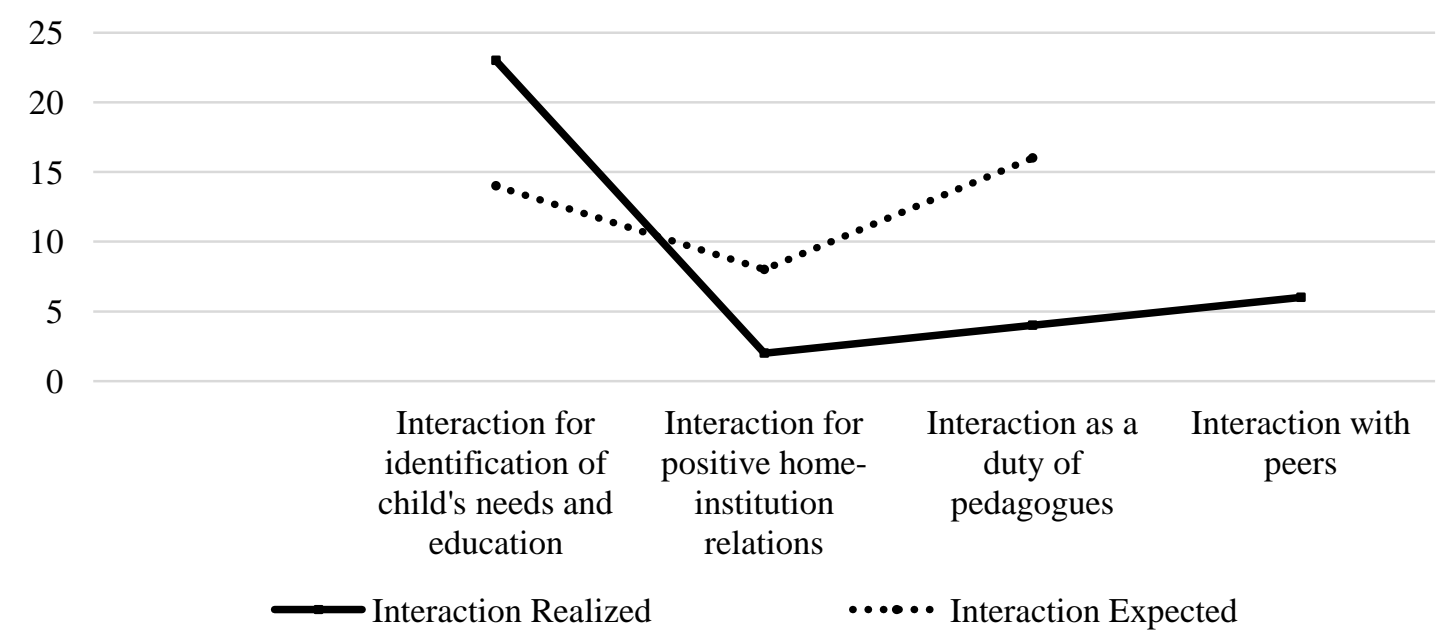

Fig. 6 Realized and expected ECEI practices related to interaction

Collaboration practices are these that promote and sustain collaborative adult partnerships, relationships, and ongoing interactions to ensure that programs and services achieve desired child and family outcomes and goals. The family is an essential member of the team and the team includes practitioners from multiple disciplines as needed (DEC, 2014). Five key categories related to collaboration have been identified in the comments $(n=87)$ of the participants: teamwork and coordination, family as a part of the team, networking with professionals and institutions beyond of the setting, and professional competence. The results show that practitioners representing multiple disciplines work together as a team to plan and implement support and services to meet the needs of children: 'specialists work in close cooperation and know about each other's discipline very well; we support and complement each other', 'we seek to solve problems together, search for the ways how to better support families'. Practitioners and families work together as a team though not systematically, and it needs to be improved: 'specialists representing diverse professions and families work together while planning individual support to a child and a family', however, 'teamwork with families is often impossible', 'parents are not motivated, they used to require...'. Evidences show that professional competence to collaborate with families is quite low and needs to be improved: 
Stefanija Alisauskiene. The Realized and Expected Early Childhood Intervention Practices in Lithuania

'we have no multi-professional team', 'lack of collaboration forms with families', 'some specialists lack the competence to cooperate with parents', etc. Realized and expected practices related to collaboration are displayed in Fig. 7.

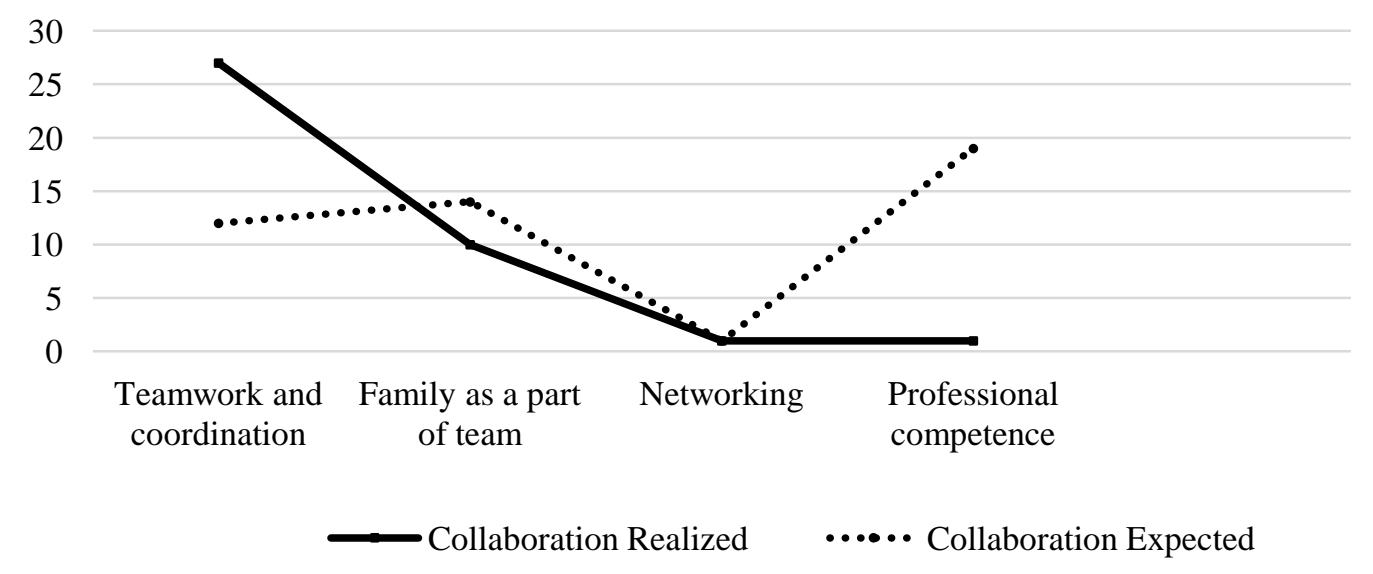

Fig. 7 Realized and expected ECEI practices related to collaboration

Transition refers to the events and processes associated with key changes between environments during the early childhood years and the practices that support the adjustment of the child and family to the new setting. These changes occur at the transition from early intervention to kindergarten and later to school. As with other life transitions or changes, positive teacher-child and practitioner-family relationships are associated with greater satisfaction, better adjustment, and better child outcomes (DEC, 2014). Analysis of the comments $(n=65)$ revealed three main categories, such as transfer of information to another setting together with a child, transfer of information to a family, transfer of information to another setting as a part of the inter-institutional collaboration. Practitioners did agree with the importance of information exchange to support positive outcomes for both child and family, and this should be a part of interinstitutional collaboration: 'we exchange information if necessary', 'specialists exchange information regarding support provided to a child when a child moves to another setting', etc.

However, the transfer of information is not a requirement in ECEI practice and this is hardly ever realized, though professionals do it voluntarily: 'there are no regulations or recommendations regarding the transfer process', 'the incoming child does not bring any documents/portfolio, sometimes parents would conceal the information related to SEN of a child'. Realized and expected practices related to transition are available in Fig. 8. 


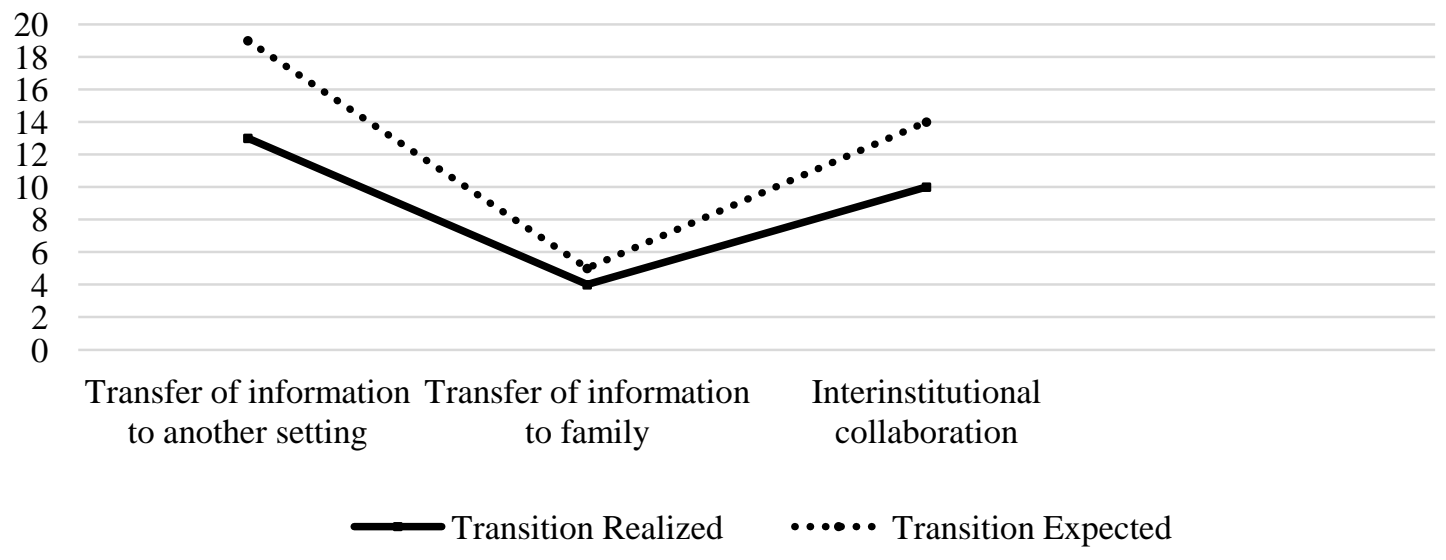

Fig. 8 Realized and expected ECEI practices related to transition

\section{Summing-up}

The categories related to realized / expected ECEI topics, such as Leadership, Assessment, Environment, Family, Instruction, Interaction, Collaboration, Transition, have been revised and the topical key categories in all analyzed topics have been selected. These are as follow: teamwork, educational environment, collaboration with families, professional competence, interinstitutional networking, and resources.

Realized ECEI practice. The results show that inter-institutional networking is most topical in the Transition, creation of available educational environment - in Interaction and Assessment, professional competence - in Instruction and Family, and collaboration with families - in Family, Assessment and Collaboration (Fig. 9).

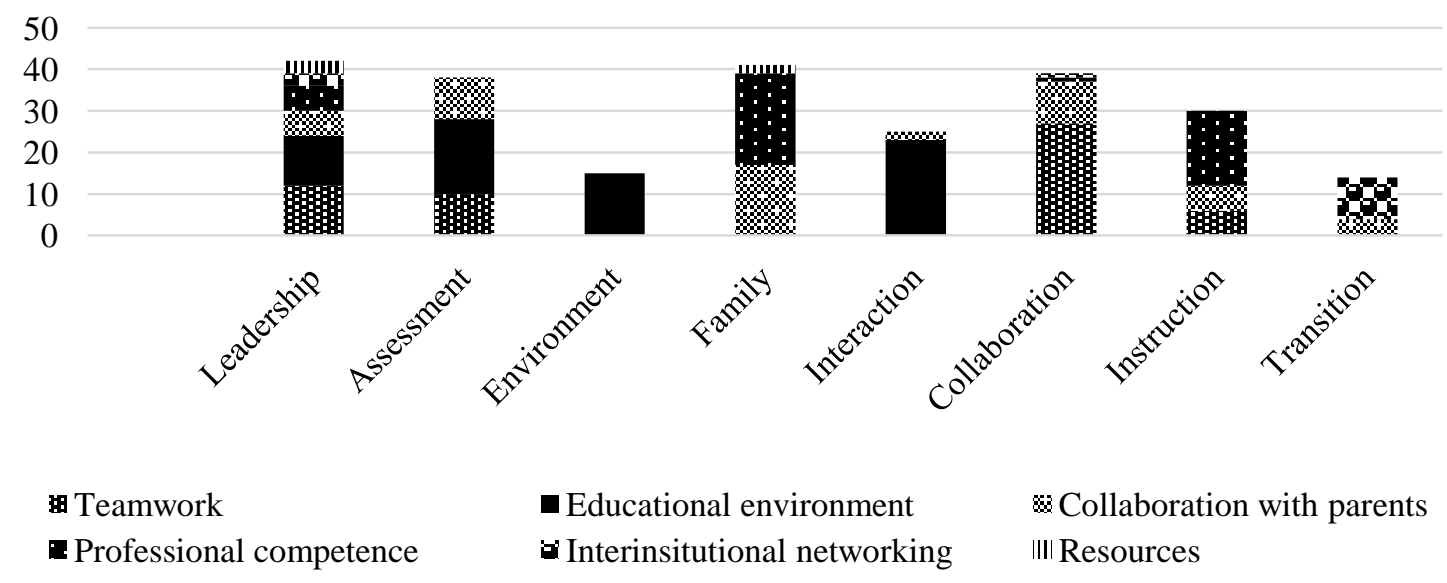

Fig. 9 Distribution of topical key categories in realized ECEI practices 
Expected ECEI practice. Evidences of the research show that teamwork is the most topical in Collaboration, professional competence - in Instruction, Collaboration, Assessment, and Family, available educational environment - in Environment and Interaction, inter-institutional networking - in Transition, collaboration with parents is the most challenging and expected to be improved in Assessment, Family, Instruction and in the rest topics of ECEI practice. Resources are the most expected for Leadership and Assessment (Fig. 10).

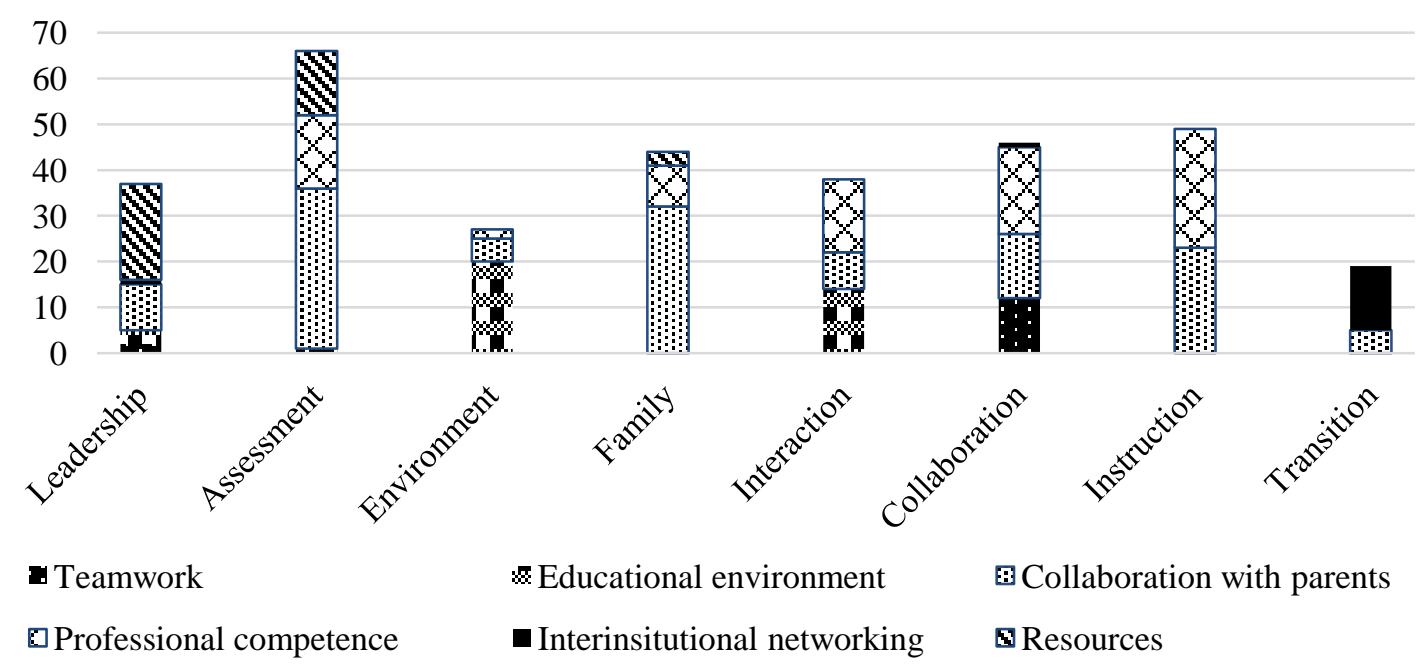

Fig. 10 Distribution of topical key categories in expected ECEI practices

\section{Conclusion}

The research showed that teaming and collaboration was the highly developed practice among ECEI specialists from multiple disciplines, though professional competence was expected to be improved, particularly for Instructional Practices, which are a cornerstone of ECEI. Teachers, other practitioners, family members, and other caregivers need to use instructional practices to maximize learning and improve developmental and functional outcomes for young children, including those with SEN.

The evidence shows that cooperation with families needs to be strengthened, particularly in Assessment, Instruction, Family, Leadership, and the rest of topics, as a family is an essential member of the team to achieve mutually agreed outcomes and goals that promote family competences and support the development of a child.

The issue of lacking resources, including financial, time, space, etc., has been highlighted as one of the main obstacles to realizing high-quality ECEI in Lithuania. The analysis of the comments revealed still existing stereotypical attitudes of ECEI specialists towards children with special educational needs 
('No children with SEN - no problem'), and misinterpretation of inclusive education ('We do not implement inclusive education because we have no children with disabilities'). The last-mentioned issue proves that all practitioners who work with young children, including those with SEN, are expected to access professional development to build knowledge and skills related to developmentally appropriate practices, discipline-specific knowledge, and positive attitudes.

\section{References}

Alghazo, E., \& Gaad, E. (2004). General Education Teachers in the UAE and Their Acceptance of the Inclusion of Students with Disabilities. British Journal of Special Education, 31 (2), 94-99.

Beach, M. (2010). Accommodations: Assisting students with disabilities. Tallahassee: Florida, Department of Education. Retrieved from http://www.learninglandscapes.ca/images/ documents/11-no14/11-no14-final-lr-links.pdf

Cate, D., Diefendorf, M., McCullough, K., Peters, M. L., \& Whaley, K. (Eds). (2010). Quality Indicators of Inclusive Early Childhood Programs/Practices. Chapel Hill: The University of North Carolina, FPG Child Development Institute, National Early Childhood Technical Assistance Center.

Conn-Powers, M., Cross, A., Traub, E., \& Hutter-Pishgahi, L. (2006). The Universal Design of Early Education: Moving Forward for All Children. Beyond the Journal - Young Children on the Web: http://journal.naeyc.org/btj/200609/ConnPowersBTJ.pdf.

Equity and Inclusive Education in Ontario Schools. Guidelines for Policy Development and Implementation. Realizing the Promise of Diversity (2014). Retrieved from http://www.edu.gov.on.ca/eng/policyfunding/inclusiveguide.pdf

Farrell, P., \& Ainscow, M. (2002). Making Special Education Inclusive. David Fulton Publisher.

Forlin, C., I., Cedillo, S., Romero-Contreras, T., Fletcher, \& Hernández, H. (2010). Inclusion in Mexico: Ensuring Supportive Attitudes by Newly Graduated Teachers. International Journal of Inclusive Education, 14 (7), 723-739.

Key Data on Early Childhood Education and Care in Europe. (2014). Retrieved from http://eacea.ec.europa.eu/education/eurydice.

Key Data on Education in Europe (2009). Retrieved from http://eacea.ec.europa.eu/ education/eurydice/documents/key_data_series/105en.pdf.

Klein, D., Cook, R. E. \& Richardson-Gibbs, A. M. (2000). Strategies for Including Children with Special Needs in Early Childhood Settings. Albany, NY: Thomson Delmar Learning.

Kozleski, E. B., Artiles, A., \& Waitoller, F. (2014). Translating Inclusive Education: Equity Concerns. In L. Florian (Eds). The Handbook of Special Education (pp. 231-249). New York: Sage Publications.

Policy Guidelines on Inclusion in Education, UNESCO (2009). Published by the United Nations Educational, Scientific and Cultural Organization, Paris, France.

Policy Statement on Inclusion of Children with Disabilities in Early Childhood Programs (2015). Retrieved from http://www2.ed.gov/policy/speced/guid/earlylearning/jointstatement -full-text.pdf 
Stefanija Alisauskiene. The Realized and Expected Early Childhood Intervention Practices in Lithuania

Talmor, R., Reiter, Sh., \& Feigin, N. (2005). Factors Relating to Regular Education Teacher Burnout in Inclusive Education. European Journal of Special Needs Education, 20 (2), 215-229.

Teacher Education for inclusion. International Literature Review (2010). Retrieved from https://www.european-agency.org/sites/default/files/te4i-international-literature-review_ TE4I-Literature-Review.pdf

Underwood, K., \& Frankel, E. (2012). The developmental systems approach to early intervention in Canada Infants \& Young Children, 25 (4), 1-11.

Woolfson, L., \& Brady, K. (2009). An Investigation of Factors Impacting on Mainstream Teachers' Beliefs about Teaching Students with Learning Difficulties. Educational Psychology, 29 (2), 221-238. 